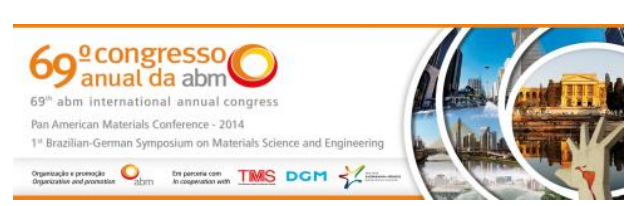

Tema: Gestão de Meio Ambiente, Recuperação e Tratamento de Rejeitos

\title{
VALORIZAÇÃO, CLASSIFICAÇÃO E CARACTERIZAÇÃO DE PCls*
}

\section{Resumo}

\author{
Barbara Mazzini Loureiro ${ }^{1}$ \\ Flávia Paulucci Cianga Silvas ${ }^{2}$ \\ Viviane Tavares de Moraes $^{3}$ \\ Jorge Alberto Soares Tenório 4 \\ Denise Crocce Romano Espinosa ${ }^{5}$
}

As placas de circuito impresso ( $\mathrm{PCl}$ ) são materiais heterogêneos e sua composição pode variar em função do ano de fabricação, tecnologia empregada, propósito de utilização, legislações vigentes e país de origem, entre outros. Essas características interferem diretamente no valor econômico agregado a estes resíduos, pois dependem da composição dos mesmos, de tal maneira que as empresas recicladoras tomam como base essas informações no momento da aquisição da sucata. Assim, a classificação e caracterização de PCls é de suma importância para o mercado de reciclagem de resíduos eletroeletrônicos. A fim de alcançar este objetivo foi realizada cominuição em moinho de facas utilizando grelhas com abertura de 9, 6 e $3 \mathrm{~mm}$. A etapa de caracterização incluiu ensaios de digestão em água régia, perda ao fogo, análises química quantitativa por ICP-OES e MEV/EDS. Verificou-se que o lote de PCI estudado possui $39,5 \%$ de material cerâmico, 34,5\% polimérico e $26 \%$ metálico. A porcentagem em massa de cobre na placa é de $14,28 \%$, de ferro $3,73 \%$, de ouro $0,012 \%$ e de estanho 1,29\%. O lote não é lead free e é multilayer com quatro camadas de cobre.

Palavras-chave: PCl; Caracterização; Reciclagem; Valor Econômico

\begin{abstract}
A printed circuit boards (PCB) is a heterogeneous material and their composition may vary depending on the year of manufacture, technology employed, purpose of use, current legislation and country of origin, among others. These characteristics directly affect the aggregate economic value of these residues, because they depend on composition thereof, so that recycling companies have built on these information at the time of waste acquisition. Therefore, the classification and characterization of PCBs are extremely important for the electronic waste recycling market. In order to achieve this goal comminution was performed in a Wiley mill using grids with opening of 9,6 and $3 \mathrm{~mm}$. The characterization stage included digestion in aqua regia testing, loss on ignition, quantitative chemical analysis by ICP-OES and SEM / EDS It was found that the lot of PCBs studied has $39.5 \%$ of ceramic material, $34.5 \%$ polymeric and $26 \%$ metallic. The percentage by weight of copper in the plate is $14.28 \%, 3.73 \%$ iron, $0.012 \%$ gold and $1.29 \%$ tin. The lot is not lead free and is multilayer with four layers of copper.
\end{abstract}

VALORIZATION, RATING AND CHARACTERIZATION OF PCBS

Keywords: PCB; Characterization; Recycling; Economic Value;

1 Graduanda, Engenharia Metalúrgica e de Materiais, Departamento de Engenharia Metalúrgica e de Materiais, Escola Politécnica, Universidade de São Paulo (USP), SP, Brasil.

2 Mestre, Engenharia Metalúrgica e de Materiais, Doutoranda em Engenharia Metalúrgica e de Materiais, Depto. de Engenharia Metalúrgica e de Materiais, Escola Politécnica, USP, SP, Brasil.

3 Doutor, Engenharia Metalúrgica e de Materiais, Pós-doutoranda em Engenharia Metalúrgica e de Materiais, Depto. de Engenharia Metalúrgica e de Materiais, Escola Politécnica, USP, SP, Brasil.

4 Doutor, Engenharia Metalúrgica e de Materiais, Prof. Titular, Departamento de Engenharia Metalúrgica e de Materiais, Escola Politécnica, USP, SP, Brasil.

5 Doutor, Engenharia Metalúrgica e de Materiais, Prof. Associado, Departamento de Engenharia Metalúrgica e de Materiais, Escola Politécnica, USP, SP, Brasil.

\footnotetext{
* Contribuição técnica ao $69^{\circ}$ Congresso Anual da ABM - Internacional e ao 14ํㅡㄹ ENEMET - Encontro Nacional de Estudantes de Engenharia Metalúrgica, de Materiais e de Minas, 21 a 25 de julho de 2014, São Paulo, SP, Brasil.
} 


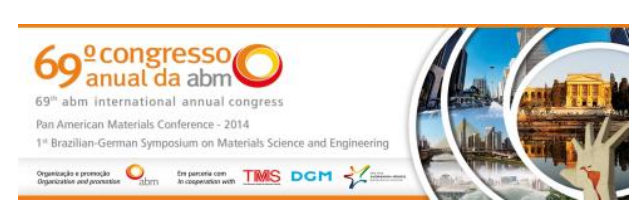

\section{INTRODUÇÃO}

Os resíduos eletroeletrônicos (REEEs) são partes ou compostos de equipamentos que são descartados por deixarem de funcionar ou por sua tecnologia se tornar obsoleta [1]. Neles há a presença de alguns materiais preciosos e outros perigosos o que alerta tanto para a possibilidade de recuperação da fração metálica quanto para os cuidados com sua disposição final. O ciclo de vida dos equipamentos eletrônicos é cada vez menor e, portanto, a quantidade de equipamentos que se tornam obsoletos em um curto espaço de tempo é crescente, assim como as preocupações relacionadas ao descarte de modo a evitar danos ao meio ambiente e à saúde pública [2]. Em 2009 a geração estimada de resíduos eletroeletrônicos no Brasil foi de 679000 t/ano. A previsão para 2030 é de 953500 t/ano [3].

As placas de circuito impresso (PCls) estão presentes em praticamente todos os equipamentos eletroeletrônicos Elas são compostas por diversos metais base como ferro, cobre, alumínio, chumbo e estanho, assim como metais preciosos. Ligas metálicas também estão inclusas. Assim pode-se dividir em cerâmicos, metálicos e poliméricos os materiais presentes nas placas e seus componentes [4].

As PCls são heterogêneas e sua composição pode variar em função do ano de fabricação, tecnologia empregada, propósito de utilização, legislações vigentes e país de origem, entre outros [5].

Os custos e problemas ambientais relacionados à disposição final das placas, assim como a escassez de recursos naturais e consequente aumento nos custos de fabricação de novos equipamentos estimulam as empresas em se preocupar com métodos alternativos de lidar com esses resíduos [6]. Assim, a reciclagem de PCls já é uma realidade em diversos países como Bélgica, onde tem-se instalada a Umicore S.A., uma das maiores empresas de reciclagem na área [7].

No entanto, no Brasil, ainda não há tecnologia consolidada para a reciclagem destes resíduos. A situação atual baseia-se num mercado repleto de intermediadores de compra e venda de "sucata", onde no final são encaminhadas para as empresas recicladoras internacionais que tomam como base para remuneração no momento da aquisição a porcentagem e o valor dos materiais que podem vir a ser recuperados. Assim, a classificação e caracterização de PCls são de suma importância para o mercado de reciclagem de resíduos eletroeletrônicos.

Impulsionado pelo crescimento do setor e visando melhor aproveitamento econômico dos metais que podem vir a ser recuperados, é preciso aperfeiçoar a separação das $\mathrm{PCls}$, que, em geral, é feita em lotes relacionados à composição química [8].

A primeira separação costuma ser feita manualmente, por catadores ou cooperativas, e é limitada pelo conhecimento prévio da composição relacionada à aparência da placa, o que resulta em frequentes falhas e prejuízos [9]. Assim, de modo a minimizar estes erros este trabalho visa caracterizar um lote de placas de circuito impresso de forma a determinar a porcentagem de materiais (cerâmico, polimérico e metálico), bem como identificar e quantificar os metais presentes e, por fim relacionar sua composição ao valor econômico agregado do lote estudado.

\footnotetext{
* Contribuição técnica ao 69ำ Congresso Anual da ABM - Internacional e ao 14ํㅡㄹ ENEMET - Encontro Nacional de Estudantes de Engenharia Metalúrgica, de Materiais e de Minas, 21 a 25 de julho de 2014, São Paulo, SP, Brasil.
} 


\section{MATERIAIS E MÉTODOS}

\subsection{Aquisição das Amostras}

Com o intuito de simular as condições aplicadas ao mercado de compra e venda de sucata eletroeletrônica separou-se para caracterização um lote de placas de circuito impresso composto por PCls provenientes de equipamentos diversos como impressoras e computadores obsoletos (figura 1). Essa separação é feita usualmente na indústria para fins comerciais devido ao valor semelhante associado a cada tipo placa.

Para processamento mecânico foram utilizados aproximadamente 300 gramas do lote.

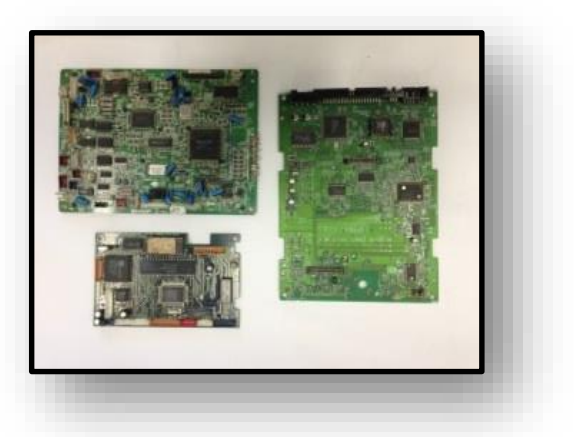

Figura 1. Placas diversas

\subsection{Processamento Físico}

As PCls foram cominuídas em um moinho de facas, passando sequencialmente a amostra por grelhas de $9 \mathrm{~mm}, 6 \mathrm{~mm}$ e $3 \mathrm{~mm}$. Essa granulometria oferece suficiente liberação dos metais para lixiviação [9].

\subsection{Caracterização Química}

\subsubsection{Lixiviação em água régia}

Separou-se para lixiviação em água régia aproximadamente $5 \mathrm{~g}$ do lote por quarteamento. Utilizou-se uma proporção de 1:20, $20 \mathrm{ml}$ de água régia para cada $1 \mathrm{~g}$ de amostra [11]. A solução permaneceu durante $24 \mathrm{~h}$ a temperatura ambiente, para completa digestão dos metais. As próximas etapas foram filtração em papel de filtro quantitativo seguido de análise do líquor por Espectrometria de Emissão Atômica por Plasma Acoplado (ICP-OES) visando quantificar os metais presentes.

\subsubsection{Lixiviação em ácido nítrico}

Os sólidos filtrados na etapa de lixiviação em água régia foram colocados em ácido nítrico $10 \mathrm{M}$ durante $2 \mathrm{~h}$ em temperatura ambiente na proporção $1: 5,5 \mathrm{ml}$ de ácido para cada $1 \mathrm{~g}$ de sólido. Utilizou-se uma solução $10 \mathrm{M}$ uma vez que a prata é solubilizada em solução $8 \mathrm{M}$ de ácido nítrico [12]. Essa etapa foi realizada com intenção de verificar se ainda existiam metais não solubilizados na etapa anterior (lixiviação em água régia). Após a filtragem do resíduo sólido em papel de filtro quantitativo, o líquor foi analisado por Espectrometria de Emissão Atômica por Plasma Acoplado (ICP-OES) visando quantificar os metais presentes.

* Contribuição técnica ao 69 Congresso Anual da ABM - Internacional e ao 14ํㅡㄹ ENEMET - Encontro Nacional de Estudantes de Engenharia Metalúrgica, de Materiais e de Minas, 21 a 25 de julho de 2014, São Paulo, SP, Brasil. 


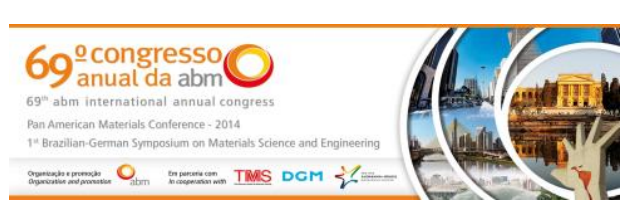

O resíduo não lixiviado foi seco em estufa, $60^{\circ} \mathrm{C}$ por $48 \mathrm{~h}$, pesado para cálculo do balanço de massa e então encaminhado para análise de perda ao fogo.

\subsubsection{Perda ao fogo}

O resíduo remanescente da lixiviação em ácido nítrico foi colocado em navícula de cerâmica previamente pesada e levado ao forno a $800^{\circ} \mathrm{C}$ por $1 \mathrm{~h}$. Após resfriamento, a navícula foi novamente pesada e, após descontar a massa da navícula, tem-se que a diferença de massa entre o resíduo inicial e final corresponde a parte orgânica que evaporou e, portanto, representa a fração polimérica presente nas placas. Já o que restou na navícula corresponde à fração cerâmica, parte inorgânica [1].

\subsubsection{Quantificação das frações}

As frações de cerâmicos, polímeros e metais foram determinadas pelas equações (1) e (2):
$\mathrm{LA}=(\mathrm{M})$
$P F=(P)$
Eq. (1)
Eq.(2)

Onde LA significa Lixiviação em água régia; PF Perda ao fogo; $M$ refere-se à fração metálica; $\mathrm{P}$ à fração polimérica. A fração cerâmica foi determinada a partir das frações M e P [12].

\subsection{Análises Micro-estrutural}

\subsubsection{Preparação da amostra}

Usando resina acrílica T1 (Risitec) para embutimento a frio, preparou-se uma amostra para análise embutindo cinco pedaços das placas, lixando e posteriormente polindo com pasta de diamante.

\subsubsection{Estereoscópio}

As amostras embutidas foram observadas em estereoscópio para verificar o número de camadas de cobre das cinco placas e analisar possíveis semelhanças ou diferenças. Além disso, pretendia-se verificar a liberação dos metais após a cominuição.

\subsubsection{MEV/EDS}

As amostras preparadas na etapa anterior foram analisadas no microscópio eletrônico de varredura (MEV- Philips FEl Inspect F50 FEG) acoplado com de Espectrômetro de Fluorescência de Raios X por Energia Dispersiva (EDS/EDX Edax Ametek) visando verificar o número de camadas e a composição das mesmas. Para tanto realizou-se análises de EDS tanto pontual quanto por mapeamento (Team).

Na figura 2 tem-se um fluxograma do processo utilizado.

\footnotetext{
* Contribuição técnica ao 69ำ Congresso Anual da ABM - Internacional e ao 14ํㅡㄹ ENEMET - Encontro Nacional de Estudantes de Engenharia Metalúrgica, de Materiais e de Minas, 21 a 25 de julho de 2014, São Paulo, SP, Brasil.
} 


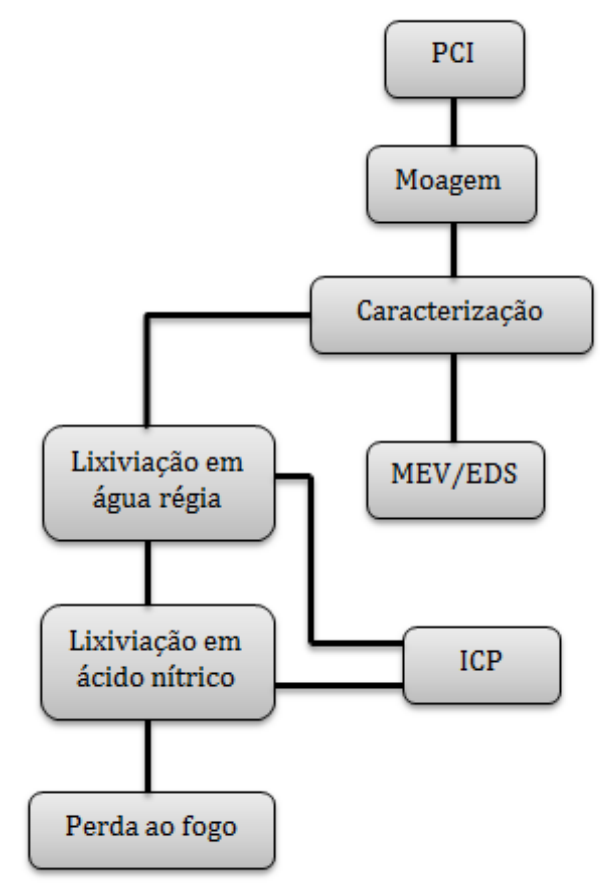

Figura 2. Fluxograma do processo

\section{RESULTADOS E DISCUSSÃO}

\subsection{Processamento Físico}

Após a moagem observou-se pequenas perdas de massa relativas ao processo, $3 \%$, comparando-se com perdas em escala industrial [13].

Verificou-se com o auxilio de estereoscópio que o material cominuido estava com sua parte metálica liberada.

\subsection{Caracterização Quimica: Lixiviação em água régia e ácido nítrico e perda ao fogo}

Após a sequência de lixiviações e ensaio de perda ao fogo foi possível determinar a composição das frações poliméricas, cerâmica e metálica do lote de PCls estudados utilizando as equações de balanço de massa. (Figura 3)

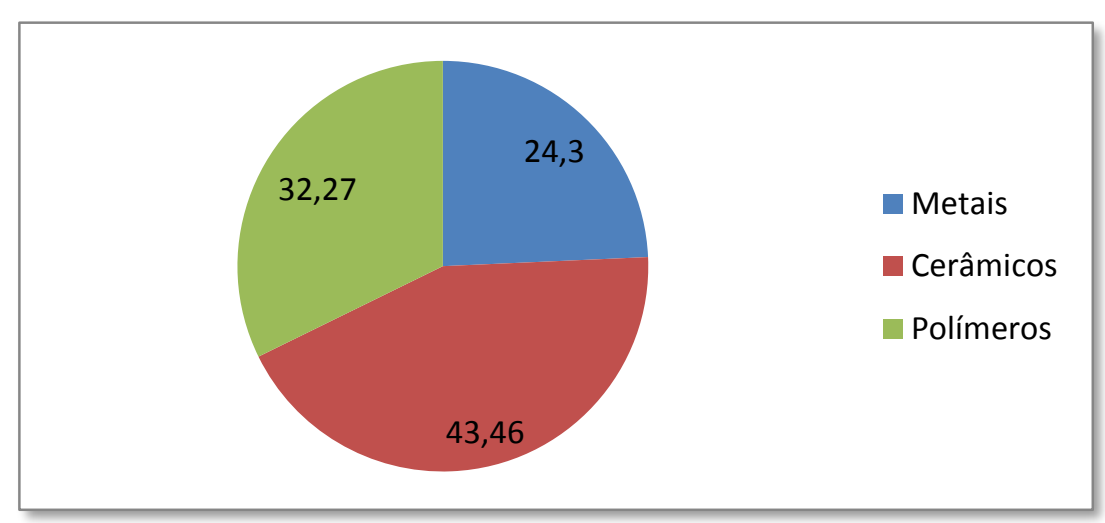

Figura 3. Gráfico de \% massa de cada fração no lote

\footnotetext{
* Contribuição técnica ao $69^{\circ}$ Congresso Anual da ABM - Internacional e ao 14ํㅡㄹ ENEMET - Encontro Nacional de Estudantes de Engenharia Metalúrgica, de Materiais e de Minas, 21 a 25 de julho de 2014, São Paulo, SP, Brasil.
} 


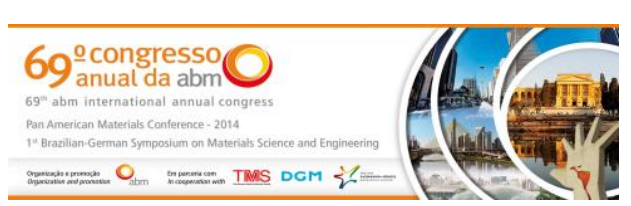

Verificou-se que o lote estudado possui $24,3 \%$ em massa de metais, $43,46 \%$ de fração cerâmica e $32,27 \%$ de fração polimérica. Segundo Yamane e Moraes et al, [13] para computadores foram encontrado $45 \%$ metais, $27 \%$ polímeros e $28 \%$ cerâmicos. Já Veit et al. [14] encontrou também para placas de computadores $40 \%$ metais $30 \%$ cerâmicos e $30 \%$ de polímeros, em média. Portanto, os resultados encontrados mostram que esse lote possui uma fração metálica menor que a média.

Opostamente ao que sugere Petter et al. [15] a lixiviação em água régia mostrou ser suficiente para solubilizar os metais presentes na placa inclusive a prata, pois a lixiviação em ácido nítrico resultou em porcentagens massa de metais menores que $0,1 \%$ e para prata não houve solubilização.

A porcentagem em massa dos metais presentes nesse lote foi calculada a partir dos resultados obtidos com a análise de ICP-OES. Os valores estão apresentados na tabela 1.

Tabela 1. \% massa de cada metal

\begin{tabular}{|c|c|}
\hline Metal & \% massa da placa \\
\hline Prata & 0,062 \\
\hline Alumínio & 3,03 \\
\hline Ouro & 0,020 \\
\hline Bário & 0,025 \\
\hline Cálcio & 0,07 \\
\hline Cobalto & 0,016 \\
\hline Cobre & 16,67 \\
\hline Manganês & 1,04 \\
\hline Níquel & 0,35 \\
\hline Chumbo & 0,87 \\
\hline Estanho & 2,93 \\
\hline Zinco & 0,84 \\
\hline
\end{tabular}

O resultado está próximo do obtido por balanço de massa $(24,3 \%$ em massa de metais) devido à heterogeneidade da amostra. Alem disso, pode haver a presença de outros metais que não foram identificados na análise química por ICP-OES.

Park e Fray [11] encontraram $0,025 \%$ de ouro, $16 \%$ de cobre,3\% de estanho, $2 \%$ de chumbo e $5 \%$ de ferro. Já Tuncuk et al.[8] encontrou $0,01 \%$ de ouro, $18,5 \%$ de cobre, $4,9 \%$ de estanho, $2,7 \%$ de chumbo e $2,1 \%$ de ferro.

Observa-se que este lote de placas continha PCls que não são lead free, pois observa-se a presença de quase $1 \%$ de chumbo. Isso era esperado já que a norma RoHS lead free, uma diretiva europeia de 2006 que proíbe que o chumbo seja usado no processo de fabricação, é relativamente recente e, algumas das placas usadas neste estudo são de computadores antigos.

Dentre os metais mais abundantes encontrados ressalta-se para o cobre, 16,67 \% em massa em relação à massa total da placa. Em 2013 o preço do cobre ficou com média de 7.926,00 U\$/t (DNPM). Considerando que haja uma recuperação de 100\% do cobre presente espera-se recuperar em $1 \mathrm{t}$ de placa, $166 \mathrm{~kg}$ de cobre, o que equivale a 1.315,72 dólares. As reservas brasileiras de minério de cobre possuem um teor médio de $0,8 \%$ de cobre (DNPM). Portanto há maior disponibilidade de cobre na placa do que no minério, o que tem influenciado o grande número de estudos na área.[1, 4, 10, 11, 13, 16]. A mesma conclusão pode ser feita para o ouro, já que as reservas brasileiras possuem um teor de ouro de 0,0002\% aproximadamente (DNPM), e uma placa possui 100 vezes mais ouro.

* Contribuição técnica ao 69ำ Congresso Anual da ABM - Internacional e ao 14ํㅡㄹ ENEMET - Encontro Nacional de Estudantes de Engenharia Metalúrgica, de Materiais e de Minas, 21 a 25 de julho de 2014, São Paulo, SP, Brasil. 
A partir da cotação na London Metals em março de 2014 calcula-se que o valor total para $100 \%$ dos metais da peça recuperados em 10.187,76 dólares.

Esses resultados confirmam o interesse na recuperação dos metais presentes na sucata, tanto pelo alto valor dos metais preciosos como pela porcentagem encontrada.

\subsection{Análises Microscópica}

\subsubsection{Análise em estereoscópio}

Utilizou-se estereoscópio para analisar as camadas das diferentes placas do lote. Verificou-se que todas as placas visualizadas apresentavam a mesma estrutura, com quatro camadas aparentes. (figura 4)
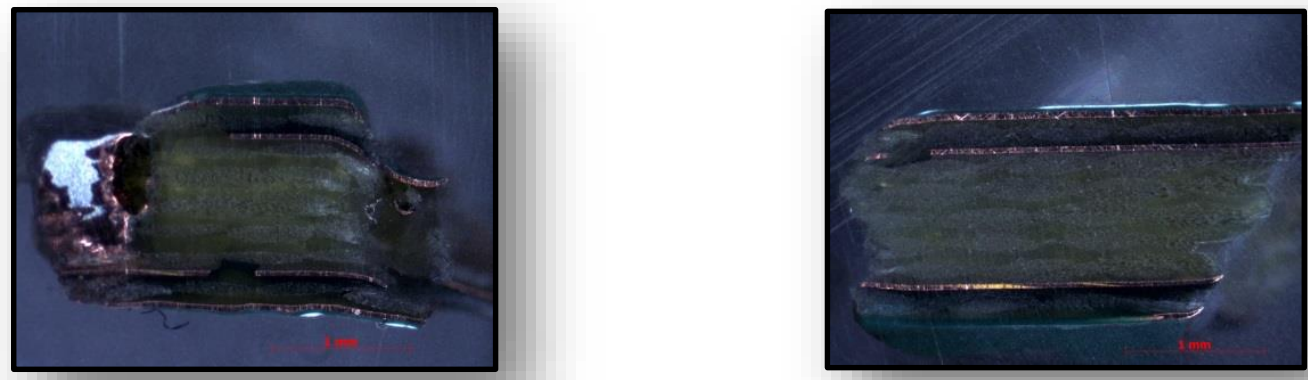

Figura 4. Fotografias no estereoscópio

Inicialmente costuma-se dividir em duas categorias, dependendo da finalidade da placa. Na primeira se incluem placas de circuito impresso analógicas RF e de microondas, de baixa complexidade. A outra abrange todas as placas de circuito impresso digital, multilayers, como de computadores e celulares [6].

\subsubsection{Análise por MEV/EDS}

Na figura 5 tem-se a micrografia de uma das 5 amostras utilizadas.

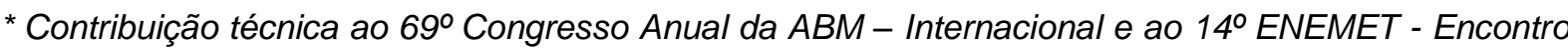
Nacional de Estudantes de Engenharia Metalúrgica, de Materiais e de Minas, 21 a 25 de julho de 2014, São Paulo, SP, Brasil.
} 

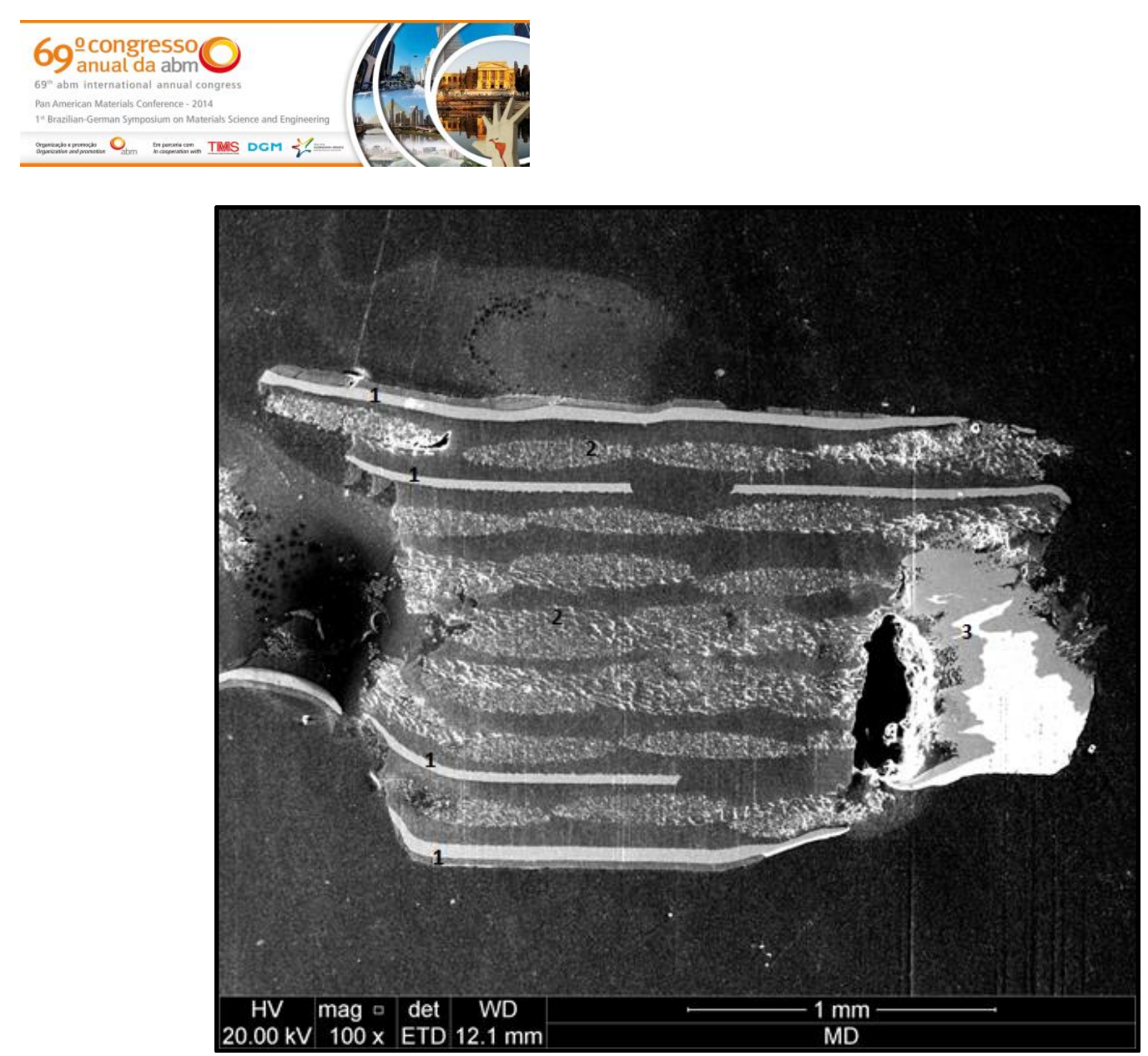

Figura 5. Fotografia MEV

As outras 4 amostras apresentaram a mesma estrutura com 4 camadas, o que corrobora com os resultados verificados no estereoscópio.

Fez-se a analise pontual de EDS para identifica a composição de cada camada, conforme figura 5.

Como esperado, as quatro camadas identificadas com o número 1 são compostas por cobre. Nas camadas identificadas com 2 verificou-se a presença de silício e cálcio, provenientes da fibra de vidro. Em 3 a parte cinza escuro é cobre, e a parte mais clara predominantemente estanho com alguns pontos de chumbo. Sendo essa parte provavelmente um ponto de contato de solda de algum componente.

Assim, verificou-se que são placas compostas de fibra de vidro, intercaladas com quatro camadas de cobre, sendo, portanto mutilayer, múltiplas camadas na placa. Resultado também encontrado por Kasper et al.2011[17]

\section{CONCLUSÃO}

A partir da analise dos resultados concluiu-se que:

- O lote possui $24,3 \%$ em massa de metais, 43,46\% de fração cerâmica e 32,27\% de fração polimérica.

- O lote estudado possui placas que não são lead free.

- As PCls estudadas são multilayers, com quatro camadas de cobre intercaladas com fibra de vidro.

- Verificou-se a presença de uma gama de metais, sendo os de maior interesse: cobre $(16,67 \%)$, estanho $(2,93 \%)$, ouro $(0,02 \%)$, alumínio $(3 \%)$, prata $(0,062 \%)$, zinco $(0,8 \%)$ e $\mathrm{Ni}(0,35 \%)$.

-Pode ser recuperado $10.187,76$ dólares por tonelada se $100 \%$ dos metais fosse recuperados.

\footnotetext{
* Contribuição técnica ao 69ำ Congresso Anual da ABM - Internacional e ao 14ํㅡㄹ ENEMET - Encontro Nacional de Estudantes de Engenharia Metalúrgica, de Materiais e de Minas, 21 a 25 de julho de 2014, São Paulo, SP, Brasil.
} 


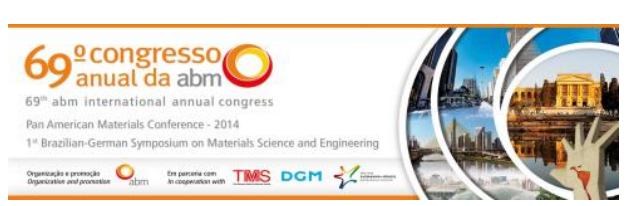

-A concentração de cobre e ouro é maior que nos minérios, influenciando estudos na área, destacando a importância da caracterização para processos futuros de reciclagem e recuperação das frações metálicas.

\section{Agradecimentos}

À FAPESP, processo 17412-0, CNPq e CAPES.

\section{REFERÊNCIAS}

1 Moraes V. Recuperação de metais a partir do processamento mecânico e hidrometalúrgico de placas de circuito impresso de celulares obsoletos. [doutorado]. São Paulo: Escola Politécnica da Universidade de São Paulo; 2011.

2 Robinson $\mathrm{BH}$. E-waste: an assessment of global production and environmental impacts. Sci. Total Environ., 2009; 408:183-191

3 Meius Engenharia. Diagnóstico da Geração de Resíduos Eletroeletrônicos no Estado de Minas Gerais. Belo Horizonte, 2009.

4 Oliveira PCF. Valorização de Placas de Circuito Impresso por Hidrometalurgia [doutorado]. Lisboa: Universidade Técnica de Lisboa, 2012.

5 Marques AC, Cabrera JM, Fraga Malfatti CF. Printed circuit boards: A review on the perspective of sustainability. Journal of Environmental Management. 2013; 131: 298306

6 Coombs CF. Printed Circuits Handbook. 6ํㅡㄹ Edição. Estados Unidos: The McGraw-Hill Companies; 2008.

7 Umicore S.A. Precious Metals Refining. 2014 [acesso em 30 mar. 2014]. Disponivel em: http://www.preciousmetals.umicore.com/PMR/.

8 Tuncuk A, Akcil A, Yazici E.Y, Devici H. Aqueous metal recovery techniques from escrap: hydrometallurgy in recycling. Miner, 2012. Eng. 25, 28-37

9 Reuter MA; Hudson C; van Schaik A; Heiskanen K; Meskers C; Hagelüken C. Metal Recycling: Opportunities, Limits, Infrastructure, A Report of the Working Group on the Global Metal Flows to the International Resource Panel. UNEP 2013

10 Zhang S, Forssberg E. Mechanical separation-oriented characterization of electronic scrap. Resources, Conservation and Recycling, 1997; 21:247-269

11 Park YJ, Fray DJ. Recovery of high purity precious metals from printed circuit boards. Journal of Hazardous Materials, 2009; 164: 1152 - 1158

12 Vogel Al. Química Analítica Qualitativa. 5a Edição. São Paulo: Mestre Jou;1992

13 Yamane LH, Moraes VT, Espinosa DCR, Tenório JAS. Recycling of WEEE: Characterization of spent printed circuit boards from mobile phones and computers. Waste Management. . 2011; 31(12): 2553-2558

14 Veit HM, Bernardes AM, Ferreira JZ, Tenório JAS, Malfatti CF. Recovery of copper from printed circuit boards scraps by mechanical processing and electrometallurgy. Journal of Hazardous Materials. 2006; B (137): 1704-1709.

15 Petter PMH, Veit HM, Bernardes AM. Evaluation of gold and silver leaching from printed circuit board of cellphones. Waste Management, 2014; 34(2): 475-482

16 Zhang S, Forssberg E. Mechanical separation-oriented characterization of electronic scrap. Resources, Conservation and Recycling, 1997; 21(4): 247-269

17 Kasper AC, Berselli GBT, Freitas BD, Tenório JAS, Andréa M. Bernardes AM, Veit HM. Printed wiring boards for mobile phones: Characterization and recycling of copper. Waste Management. 2011; 31(12): 2536-2545.

\footnotetext{
* Contribuição técnica ao 69ำ Congresso Anual da ABM - Internacional e ao 14ํㅡㄹ ENEMET - Encontro Nacional de Estudantes de Engenharia Metalúrgica, de Materiais e de Minas, 21 a 25 de julho de 2014, São Paulo, SP, Brasil.
} 